\title{
LA-UR-09. 04891
}

Approved for public release:

distribution is unlimited.

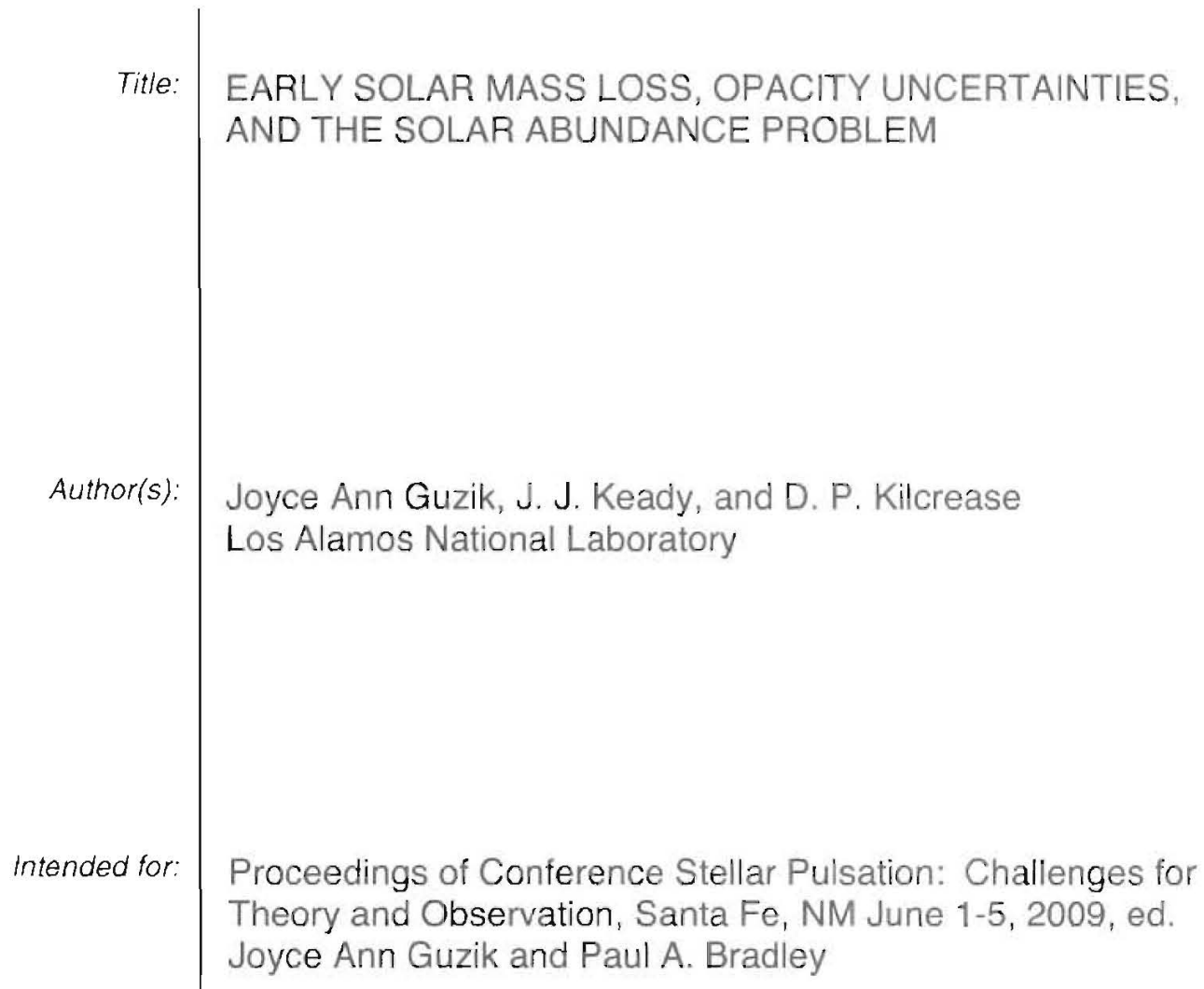

Los Alamos

NATIONAL LABORATORY

EST. 1943

Los Alamos National Laboratory, an affirmative action/equal opportunity empioyer, is operated by the Los Alamos National Security, LLC for the National Nuclear Security Administration of the U.S. Department of Energy under contract DE-AC52-06NA25396. By acceptance of this article, the publisher recognizes that the U.S. Government retains a nonexclusive, royalty-free license to publish or reproduce the published form of this contribution, or to allow others to do so, for U.S. Government purposes. Los Alamos National Laboratory requests that the publisher identify this article as work performed under the auspices of the U.S. Department of Energy. Los Alamos National Laboratory strongly supports academic freedom and a researcher's right to publish; as an institution, however, the Laboratory does not endorse the viewpoint of a publication or guarantee its technical correctness.

Form $836(7 / 06)$ 



\title{
Early Solar Mass Loss, Opacity Uncertainties, and the Solar Abundance Problem
}

\author{
Joyce Ann Guzik ${ }^{1}$, J.J. Keady ${ }^{2}$, and D.P. Kilcrease ${ }^{2}$ \\ 'X-2-N2, MS T086, Los Alamos National Laboratory, Los Alamos, NM 87545-2345 U.S.A. \\ ${ }^{2} T-1$, MS B2 14, Los Alamos National Laboralory, Los Alamos, NM 875+5-.23+5 U.S.A.
}

\begin{abstract}
Solar models calibrated with the new element abundance mixture of Asplund et al, published in 2005 no longer produce good agrcement with the sound speed, convection zone depth, and convection zone helium abundance infered from solar oscillation data. Attempts to modify the input physics of the standard model. for example, by including enhanced diffusion, increased opacities, accretion. convective overshoot, or grayity waves have not restorcd the good agreement attained with the prior abundances. Here we present new models including early mass loss via a stronger solar wind. Early' mass loss has been investigated prior to the solar abundance problem to deplete lithium and resolve the "faint early sun problem". We find that mass loss modifies the core structure and deepens the convection zone, and so improves agreement with oscillation data using the new abundances; however the amount of mass loss must be small to avoid destroying all of the surface lithium, and agreement is not fully restored. We also considered the prospects for increasing solar interior opacities. In order to increase mixture opacities by the $30 \%$ required to mitigate the abundance problem. the opacities of individual elements (e.g., O, N, C. and Fe) must be revised by a factor of two to three for solar interior conditions: we are investigating the possibility of broader calculated line wings for bound-bound transitions at the relevant temperatures to enhance opacity. We find that including all of the elements in thi: AGS05 opacity mixture (through uranium at atomic number $Z=92$ ) instead of only the 17 elements in the OPAL opacity mixture increases opacities by a negligible $0.2 \%$.
\end{abstract}

Keywords: Sun: oscillations: Sun: abundances: helioseismology; mass loss; solar wind PACS: 96.60.Ly; 96.60-j, 97.10.Tk. 97.10.Me

\section{INTRODUCTION}

The Asplund et al. (AGS05) [1] solar abundance determination revises downward the mass fraction of elements $Z$ heavier than hydrogen $(X)$ and helium $(Y)$, particularly the abundances of oxygen, carbon, and nitrogen. For the older (e.g. Grevesse and Sauval 1998; [2]) abundances, $Z / X=0.023$, and $Z \sim 0.018$. For the new abundances, $Z / X=0.0165$, and $Z$ 0.0122 . Solar models evolved with the new abundances give worse agreement with helioseismic constraints. In particular, the models with the new abundances have a $1.4 \%$ discrepancy in sound speed, too shallow a convection-zone depth, and too-low surface helium abundance. How can th is discrepancy be resolved? Should we adopt the new abundances?

Here we review the problems for the solar model with the new abundances, some of the solutions investigated to date, and investigate early mass loss via a stronger solar wind as a means to mitigate the abundance problem. See [3], [4] for some recent reviews. We also comment on opacity uncertainties and prospects for opacity enhancements to resolve the problem.

\section{CALIBRATED SOLAR MODELS WITH THE OLD AND NEW ABUNDANCES}

Solar models have many assumptions, and ingredients that are being re-examined in light of the abundance problem. Solar models require physics input, e.g., opacities, equation of state, nuclear reaction rates, convection treatment, and diffusive element settling treatment. Standard solar models are usually constructed in one-dimension, and neglect rotation, magnetic fields, mass loss, accretion, effects of waves, and convective overshooting. Standard models assume that the mass of the sun has remained constant, and that the sun began its life on the pre-main sequence with a homogeneous composition. Solar models are calibrated by adjusting the initial helium abundance and mixing length to pressure scale height ratio to match the observed luminosity, radius, and surface $\mathrm{Z} / \mathrm{X}$ at the present solar age. 
Table I summarizes our model calibrations for the Grevesse and Noels (GN93) [5] mixture and the new AGS05 mixture. For our models, we adopt the Lawrence Livermore National Laboratory OPAL opacities [6], Ferguson et al. 2005 [7] low-temperature opacities, the SIREFF analytical equation of state [8], the NACRE [9] nuclear reaction rates, and the BohmVitense [10] mixing-length theory of convection. We include thermal, gravitation and chemical diffusion of $\mathrm{H}, \mathrm{He}, \mathrm{C}, \mathrm{N}, \mathrm{O}, \mathrm{Ne}$, and using the Burgers [11] treatment as implemented by Cox, Guzik, and Kidman [12].

Table 1 also gives the helioseismic inferences for the surface $\mathrm{Y}$ and convection zone depth of Basu and Antia [13]. As is evident, models calibrated with the new mixture have too-low surface $\mathrm{Y}$ and too-shallow envelope convection zone (CZ), while the models with the GN93 abundances are in good agreement with the inferences for these quantities.

TABLE 1. Properties of calibrated standard solar models

\begin{tabular}{lcc}
\hline Model Property & GN93 Mixture & AGS05 Mixture \\
\hline Initial Y & 0.2703 & 0.2570 \\
Initial $Z$ & 0.0197 & 0.0135 \\
Mixing length to & 1.7698 & 1.9948 \\
pressure seale & & \\
height ratio $\alpha$ & & \\
$\mathrm{CZ} \mathrm{Y}$ & 0.2418 & 0.2273 \\
$\mathrm{CZ}$ base radius & 0.7133 & 0.7306
\end{tabular}

$\left(\mathrm{R}_{\text {sun }}\right)$

* Seismically-inferted CZ Y abundance and CZ base radius are $0.248 \pm 0.003$ and $0.713 \pm 0.001 R_{\text {sun }}$, respectively [13].

Figure 1 shows the sound speed profiles for the old and new abundances for the two models, using the inference of Basu et al. [14]. The error bars are about the width of the lines. While the agreement isn't perfect even for the old abundances, the new abundances give a $1.4 \%$ discrepancy just below the CZ.

A non-comprehensive list of modifications to the solar model investigated to mitigate this problem include: Increased opacity below convection zone (e.g., $[15,16]$ ); increased abundances, including enhanced Ne abundance, given uncertainties (e.g., [17, 18]); enhanced diffusive settling rates of $1.5 \mathrm{x}$ or mor: (e.g., $[13,19,20,21]$ ); accretion of lower- $Z$ material at the solar surface $[4,22]$; convective overshoot $[4]$; including the effects gravity waves below the CZ [23]. To date none of these solutions has been entirely satisfactory.

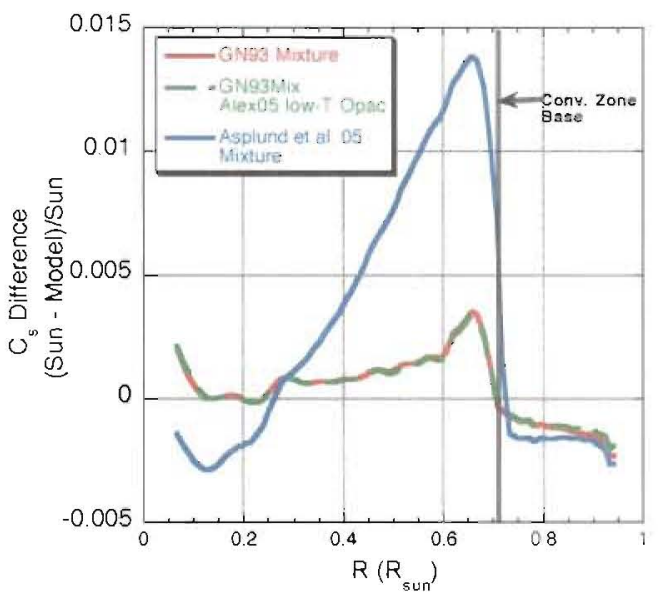

FIGURE 1. Inferred minus calculated sound-speed differences for calibrated standard one solar mass models using the GN93 and AGS05 abundances. Inferences from Basu et al. [14].

\section{MODELS WITH EARLY MASS LOSS}

Models with early mass loss were explored previously [24], [25], [26], [27], using the older higher element abundances. Minton and Malhotra [28] recently assessed consequences for Earth climate and solar system formation. The advantages of an early mass loss phase include: solving the faint early sun problem, explaining early liquid water on Mars, early inner solar system bombardment, and solar lithium destruction. Such mass loss in other stars also could potentially explain blue stragglers, and the earlierthan-predicted dredge-up of carbon and nitrogen in solar mass stars ascending the first red giant branch [29].

However, there are also drawbacks; if the sun remains at too high a mass for too long, all surface $\mathrm{Li}$ is destroyed, too much surface ${ }^{3} \mathrm{He}$ is produced, and discrepancies with the inferred sound speed arise [29].

Since mass loss hadn't been reinvestigated in light of the new abundances, we thought it would be worthwhile to do so here.

We evolved two models with initial masses 1.3 and 1.15 solar masses, having exponentially-decaying mass loss rates with e-folding time $0.45 \mathrm{Gyr}$. The initial mass loss rates were 6.55 and $3.38 \times 10^{-10}$ solar masses/year for the two models, respectively. Table 2 summarizes the initial $Y$ and mixing-length parameter needed to calibrate the models, and the final $C Z Y$ and base radius. Figure 2 shows the luminosity vs, time for these models, as well as for two constant one solarmass calibrated models. The present solar mass-loss rate is $2 \times 10^{-14}$ solar masses/year, too small to affect the sun's evolution. 


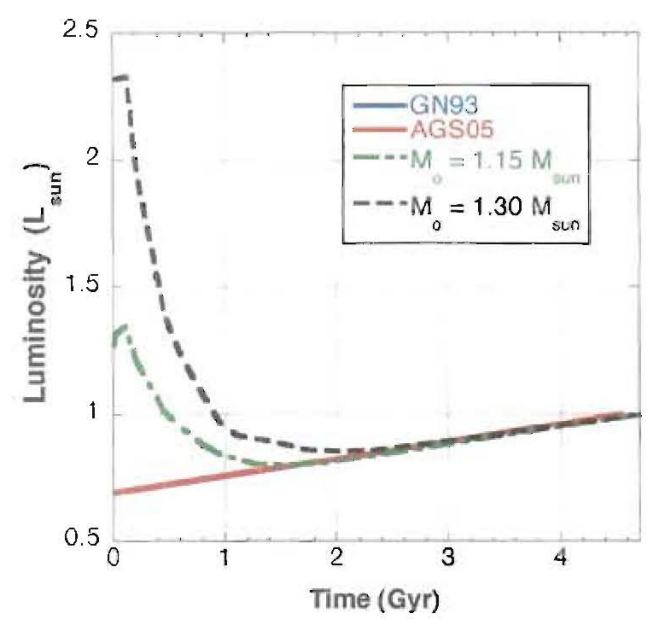

FIGURE 2. Luminosity vs, time for standard one solarmass models using the GN93 and AGS05 abundances, and for two mass-losing models using the AGS05 abundances with initial mass 1.3 and $1.15 \mathrm{M}_{\text {sun }}$. Mass-loss rates are exponentially decaying with e-folding time 0.45 Gyr.

Figure 3 shows the inferred minus calculated sound speed for these models. For the models with the AGS05 abundances, the sound speed agreement is considerably improved by including early mass loss. For the model with initial mass $1.3 \mathrm{M}_{\text {sun }}$ sound-speed agreement is almost restored near the $\mathrm{CZ}$ base, but the agreement is not as good in the more $\mathrm{H}$-depleted core. Unfortunately, while the model with initial mass 1.15 $\mathrm{M}_{\text {sun }}$ has a little better sound speed agreement in the central $0.1 R_{\text {sun }}$, the improvement is not as pronounced for the region below the $\mathrm{CZ}$.

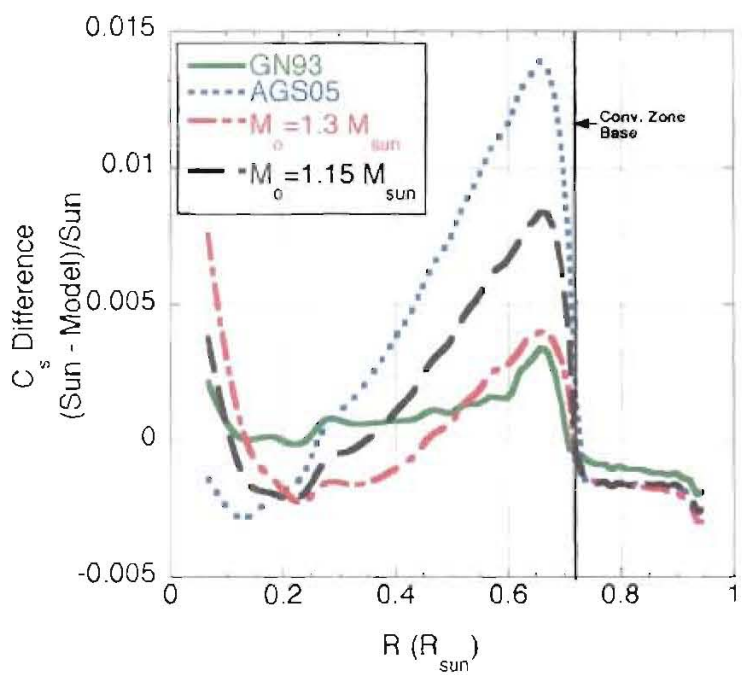

FIGURE 3. Inferred minus calculated sound speed differences for calibrated standard one solar mass models using the GN93 and AGS05 abundances, and for models with AGS05 abundances and initial mass 1.3 and $1.15 \mathrm{M}_{\text {sun }}$ including early mass loss.

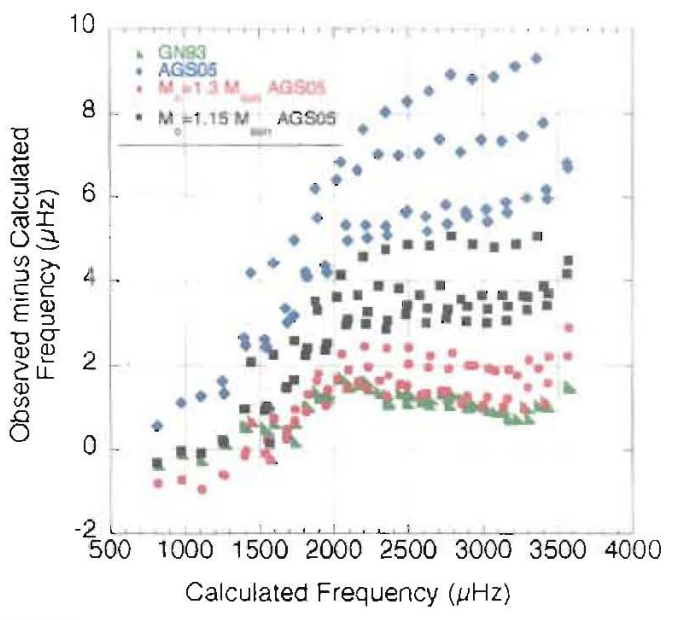

FIGURE 4. Observed minus calculated vs. calculated frequencies for calibrated standard one solar mass models using the GN93 and AGS05 abundances. and for models with AGSO5 abundances and initial mass 1.3 and $1.15 \mathrm{M}_{\text {in }}$ including early mass loss. Frequencies compared are for modes of angular degrees 0. 2, 10, and 20. Data is from [30. 31, 32]. Including mass loss improves agreement. but models with old abundances and no mass loss still give the best agreement.

TABLE 2. Properties of calibrated mass-losing models with AGS05 mixture

\begin{tabular}{lcc}
\hline Model Property & $\mathbf{M}_{0}=\mathbf{1 . 3} \mathbf{M}_{\text {sun }}$ & $\mathbf{M}_{0}=\mathbf{1 . 1 5} \mathbf{M}_{\text {sun }}$ \\
\hline Initial Y & 0.2450 & 0.2510 \\
Initial Z & 0.0135 & 0.0135 \\
Mixing length to & 2.0785 & 2.0502 \\
pressure scale & & \\
height ratio $\alpha$ & & \\
$\mathrm{CZ} \mathrm{Y}$ & 0.2324 & 0.2297 \\
$\mathrm{CZ}$ Base Radius & 0.7195 & $0.723 \mathrm{I}$ \\
$\left(\mathrm{R}_{\text {sun }}\right.$ ) & &
\end{tabular}

*Seismically-inferted $C 7 . \mathrm{Y}$ abundance and $\mathrm{CZ}$ base radius are $0.248 \pm 0.003$ and $0.713 \pm 0.001 R_{\text {sum. }}$ respectively [13].

Figure 4 shows the observed minus calculated vs. calculated nonadiabatic frequencies for modes of angular deg.ces $0,2,10$, and 20 that propagate into the solar interior below the convection zone.

The mass-losing models described here would probably destroy all of the observed surface lithium, as can be seen from Fig. 5. Lithium is destroyed in the solar interior at temperatures of $>2.8$ million $\mathrm{K}$. For standard models, on the main sequence the surface layers are never mixed to high enough temperatures to deplete $\mathrm{Li}$ by the observed factors of 200 from the initial solar system abundance, and additional mixing mechanisms must be invoked. However, with mass loss, layers that are now at the surface were initially in the interior at temperatures high enough to quickly destroy Li.

The mass-losing models also produce more ${ }^{3} \mathrm{He}$ at the surface as the now-surface layers were once 
processed at higher interior temperatures where $3 \mathrm{He}$ builds up to higher equilibrium values. For the 1.3 $\mathrm{M}_{\text {sun }}$ initial mass model, the surface ${ }^{3} \mathrm{He}$ mass fraction is enhanced from its initial value of $5 \times 10^{-5}$ to $9 \times 10^{-5}$.

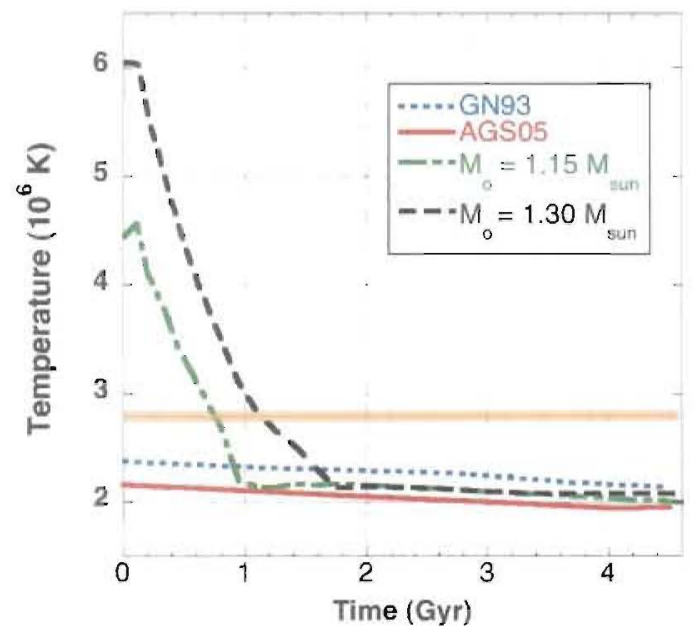

FIGURE 5. Temperature experienced by the present-day solar surface layer as a function of time for the mass losing and standard models. This temperature is attained either because the layer once resided deeper inside the sun, or because envelope convection mixes surface layers down to regions with this temperature. The horizontal line at 2.8 million $\mathrm{K}$ shows the temperature required for relatively rapid Li destruction.

\section{OPACITY UNCERTAINTIES}

Several groups, most recently ChristensenDalsgaard et al. [16], find that opacity increases of 10$30 \%$ below the convection zone are required to restore sound-speed agreement, However, the presently opacity tables from three separate projects for conditions below the $\mathrm{CZ}$ differ by only a few percent [33], [34], making it difficult to justify such large opacity enhancements.

Using the Los Alamos National Laboratory T-4 opacity library data [35], we find that, to obtain a $30 \%$ opacity increase with the new abundances, the contribution from oxygen alone would need to increase by a factor of two to three. Alternatively, the Fe absorption contribution would need to increase by a factor of three.

Inclusion of additional elements has a negligible effect. The Lawrence Livermore OPAL opacities for the AGS05 mixture included 17 of the most abundant elements. Inclusion of all of the elements up to atomic number $Z=30$ increases the mixture opacity by only $0.2 \%$ for solar interior conditions. Inclusion of additional element from $30<Z<93$, an 83-element mixture, further increases the mixture opacity by less than $0.1 \%$.
We are investigating the possibility of errors in current opacity calculations. The floor in the oxygen absorption at $1<\mathrm{hv} / \mathrm{kT}<3$, is set by L-shell photoabsorption and bremsstrahlung. However, starting above about hv/kT $=4$, the $\mathrm{K}$-shell resonance lines (their red wings in particular) creat a trough in the total oxygen absorption at about $800 \mathrm{eV}$ for temperature and density conditions typical of the solar $\mathrm{CZ}$ base, $\mathrm{T}=200 \mathrm{eV}-2.3$ million $\mathrm{K}$ and density 0.28 $\mathrm{g} / \mathrm{cc}$ (see Fig. 6). If this trough is not as deep as calculated, the Rosseland mean opacity, which has the largest weight at about this energy, $3.8 \mathrm{hv} / \mathrm{kT}$ or 800 $\mathrm{eV}(800 \mathrm{eV})$, could be affected. The same correction would also need to be made for the opacities of the other abundant elements, $\mathrm{C}, \mathrm{N}$, and Ne. Together, the $\mathrm{O}, \mathrm{C}, \mathrm{N}$, and Ne absorption would have to increase by a factor of two to affect the mixture opacity by $30 \%$.

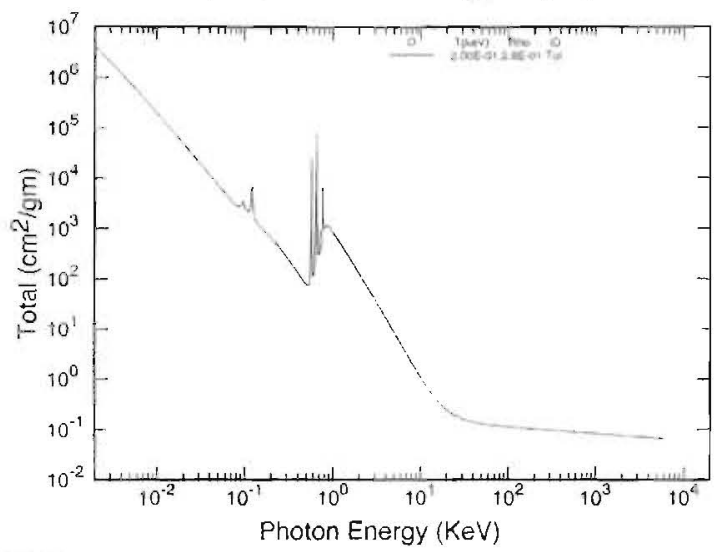

FIGURE 6. Oxygen frequency-dependent opacities form LANL T-4 Opacity Data Tables. for temperature and density conditions near the $\mathrm{CZ}$, base, $\mathrm{T}=0.2 \mathrm{keV}$, and $\rho=0.28 \mathrm{~g} / \mathrm{cc}$. An uncertainty in the trough ncar $0.8 \mathrm{keV}$ could affect the Rosseland mean opacity for this temperature with largest weight at $h v / k T \sim 3.8$.

\section{CONCLUSIONS AND FUTURE WORK}

Mass losing models can improve seismic agreement for the new abundances, but do not fully restore agreement. A smaller amount of mass loss than considered here, that leads to destruction of some, but not all of the initial lithium, could provide a plausible partial mitigation of the solar abundance problem.

Increasing the number of elements in the mixtures used for opacity calculations only negligibly increases opacities.

An as-yet unidentified error in calculations of the line wings for K-shell transitions in $\mathrm{O}, \mathrm{C}, \mathrm{N}$, and $\mathrm{Ne}$ near $800 \mathrm{eV}$ could provide some increase in the calculated Rosseland mean opacities. 
It would be advantageous to confirm theoretical opacity calculations by experiments, assuming that enough accuracy can be attained to constrain the calculations. Turck-Chièze et al. [36] discuss prospects to pursue experiments for solar and stellar interiorrelevant conditions at lasers and pulsed-power facilities.

\section{ACKNOWLEDGMENTS}

It is a pleasure to acknowledge S. Watson for prior solar model results, and J. Christensen-Dalsgaard and $\mathrm{S}$. Turck-Chiéze for many useful discussions.

\section{REFERENCES}

I. M. Asplund, N. Grevesse and A.J. Sauval, ASPC 336, 25 (2005) $\{$ AGSO $\}$.

2. N. Grevesse and A.J. Sauval, Spa. Sci. Rev. 85, 161 (1998) \{GS98\}.

3. S. Basu and H. Antia, "Helioseismology and Solar Abundances," in Physics Reports 457, $217-283$ (2008)

4. J.A. Guzik. "Problems for the Standard Solar Model Arising from the New Solar Mixture," Mem. Soc. Astron. Ital. 79,481 (2008)

5. N. Grevesse and A. Nocls, in Origin and Evolution of the Elements, Cambridge U. Press, 15 \{GN93\}.

6. C. Iglesias and F.J. Rogers, Astrophys. J. 464, 943 (1996)

7. J.W. Ferguson, D.R. Alexander, F. Allard, T. Bamnan, J. Bodnarik. P.H. Hauschildt. A. [Teffncr-Wong and A. Tamanai, Astrophys. J. 623, 585 (2005).

8. J.A. Guzik and F.J. Swenson. Astrophys. J. 491, 967 (1997).

9. C. Angulo et al, Nucl Phys. A 656, 3 (1999).

10. E. Bohm-Vitense, Zeitschrift fur Astrophysik 46, 108 (1958).

11. J.M. Burgers, in Flow Equations for Composite Gases, New York: Academic (1969).

12. A.N. Cox, J.A. Gurik and R.B. Kidman, Astrophys. J. 342, 1187 (1989).

13. S. Basu and H.M. Antia, H.M., Astrophys. J. Letl. 606 . L.85 (2004)

14. S. Basu. M.H. Yinsonneault, and J.M. Bahcall, Astrophys. J. $529,1084(2000)$

15. J.N. Bahcall, S. Basu, M.H. Pinsonneault and A.M. Serenelli, Astrophys. J. 618, 1049 (2005).

16. J. Christensen-Dalsgaard, M. Pia Di Mauro, G. Houdek and F. Pijpers, "On the opacity change required to compensate for the revised solar composition." Astron. Astrophys. 494. 205-208 (2009).

17. J.N. Bahcall, S. Basu and A.M. Serenelli, Astrophys. J. 631, 1281 (2005).

18. S. Turck-Chieze, S., Couvidal and L. Pia in EAS Publication Series 17. 149 (2005).

19. J.A. Guzik. L.S. Watson and A.N. Cox, Astrophys. J. 627, $1049(2005)$.

20. W.M. Yang and S.L. Bi, Astrophys. J. Lett. 658, L67 (2007).

21. J. Montalban, A. Miglio, A. Noels, N. Grevesse and M.P. Di Mauro, in Helio- and Asteroseismology: Towards a
Golden Furure, proc. SOHO14/GONG2004 Workshop. ed. D. Danesy, ESA SP-559, 574 (2004).

22. M. Castro, S. Vauclair and O. Richard, Astron. Astrophys. 463, 755

23. D. Amett, private communication (2006) as reported by J.A. Guzik, "Reconciling the revised solar abundances with helioscismic constraints," Proc. SOHO18/GONG2006/HELAS I, Sheffield. UK, ESA Sp-624, 17.1 (2006).

24. J.A. Guzik, L.A. W'illson and W. Brunish, Astrophys. J. 319.957-965 (1987).

25. F.J. Swenson and J. Faulkner, Astrophys. J. 395, 654 (1992).

26. J.A. Guzik and A.N. Cox, Astrophys. J. 448, 905 (1995).

27. I.-J. Sackmann and A.I. Boothroyd, Astrophys. J. 583, 1024-1039 (2003).

28. D.A. Minton and R. Malhotra, "Assessing the massive young sun hypothesis to solve the warm young Earth puzzle". Astrophys. f. 660, 1700-1706 (2007).

29. J.A. Guzik, "Effects of Main Sequence Mass Loss on Stellar and Galactic Chemical Evolution,"Ph.D. Thesis. lowa State University. Amcs, lowa (1988).

30. W.J. Chaplin. Y. Elsworth. G.R. Isaak. R. Lines, C.P. McLeod, B.A. Miller, B.A. and R. New. Mon. Not. Rov. Astron. Soc. 300, 1977 (1998).

3!. I. Schou and S. Tornczyk, $m 2$ table, hitp: //www.hao.ucar.edu/public/research/miso//,owL/data.ht $\mathrm{ml}$ (1996).

32. R.A. Garcia et al. Sol. Phys. 200, $361-379$ (2001).

33. C. Neuforge-Verheecke, J.A. Guzik. J.I. Keady, N.II. Magee, P.A. Bradley and A. Noels, Astrophys. J. 561. $450(2001)$

34. N.R. BadnclI. M.A. Bautista, K. Butler. F. Delahaye, C. Mendoza P. Palmeri, C.J. Zeippen and M.I. Seaton, Mon. Not. Roy. Astron. Soc. 360.458 (2005).

3j. N. Magee et al.. ASP Conf. Ser.78, 51 (1995)

36. S. Turck-Chieze, F. Delahaye, D. Gilles, G, Loisel and L. Piau, "On plasma radiative properties in stellar Conditions," preprint submitted to High Energy Densily Physics, June 1, 2009. 
